\title{
Diatomite as a potential drug carrier for Itraconazole and improvement in release control
}

Roufaida Merir ${ }^{1, *}$, Milad Baitiche', Ferhat Djerboua ${ }^{1}$, Mokhtar Boutahala ${ }^{2}$

${ }^{1}$ Laboratoire de Préparation, Modification et Applications des Matériaux Polymériques Multiphasiques, Département de génie des procédés, Faculté de technologie,

Université Ferhat Abbas Sétif1

${ }^{2}$ Laboratoire de Génie des Procédés Chimiques, Département de génie des procédés, Faculté de technologie, Université Ferhat Abbas Sétifl

A Abstract:

Many obstacles associated with the use of conventional drug delivery systems have led to the development of new various micro/nano sized drug carriers. These carriers are designed with the aim to improve therapeutic outcomes and/or reduce drug's adverse effects, by providing protection of the entrapped drug against in vivo degradation, releasing drug in desired man-ner, improving drug solubility and/or reducing its immunogenicity. Additionally, their small sizes make these carriers when loaded with the bioactive molecules suitable and allowing their vectorization to the target site. In this work, Diatomite (DTM) was used as a support material for Itraconazole (ITZ) which is known for its fairly low side effects. The major drawback in the therapeutic application and efficacy of ITZ as oral dosage forms is its very low aqueous solubility. Three Binary systems were prepared using different proportions of the two components and where tested for the ability of DTM to improve the solubility of ITZ in aqueous and in organic media. The efficacy of encapsulation was demonstrated by UV analysis. The prepared systems were characterized using UV-Vis, FTIR, MEB, AFM and Optical microscopy. Moreover, the study of kinetics and mechanism of drug release in the gastric medium exhibit a sustained profile during 02 hours.

Introduction Today smart technologies are used in several domains. Most of them are related with pharmaceutical industry and biomedical applications. The most recent are poorly soluble drug in aqueous solvents, that's why the modern medicine address a formidable challenge to resolve this hurdle by developing a new drug delivery systems. The main objective is to enhance the solubility, the drug efficiency and minimized the side effects. Itraconazole is hydrophobic agent having low side effects and widely prescribed for normal and immunocompromised hosts with serious fungal infections. [1]

Therefore, in the last years, emerging natural porous materials for biomedical applications have also been suggested to overcome the shortcomings of the synthetic porous materials like Diatomite. This magic bullet are characterized by excellent biocompatibility, nontoxicity and thermal stability[2]. The aim of this research was to enhance the solubility capacity of itraconazole using pure Diatomite silica and the modified one with several techniques(calcination and chemical methods) as encapsulation system. The efficacy of encapsulation was demonstrated by standard methods such as extraction and UV analysis. The prepared systems were characterized using UV-Vis, FTIR, MEB, AFM and Optical microscopy. Moreover, the study of kinetics and mechanism of drug release in the gastric medium exhibit a sustained profile during a time of two hours.

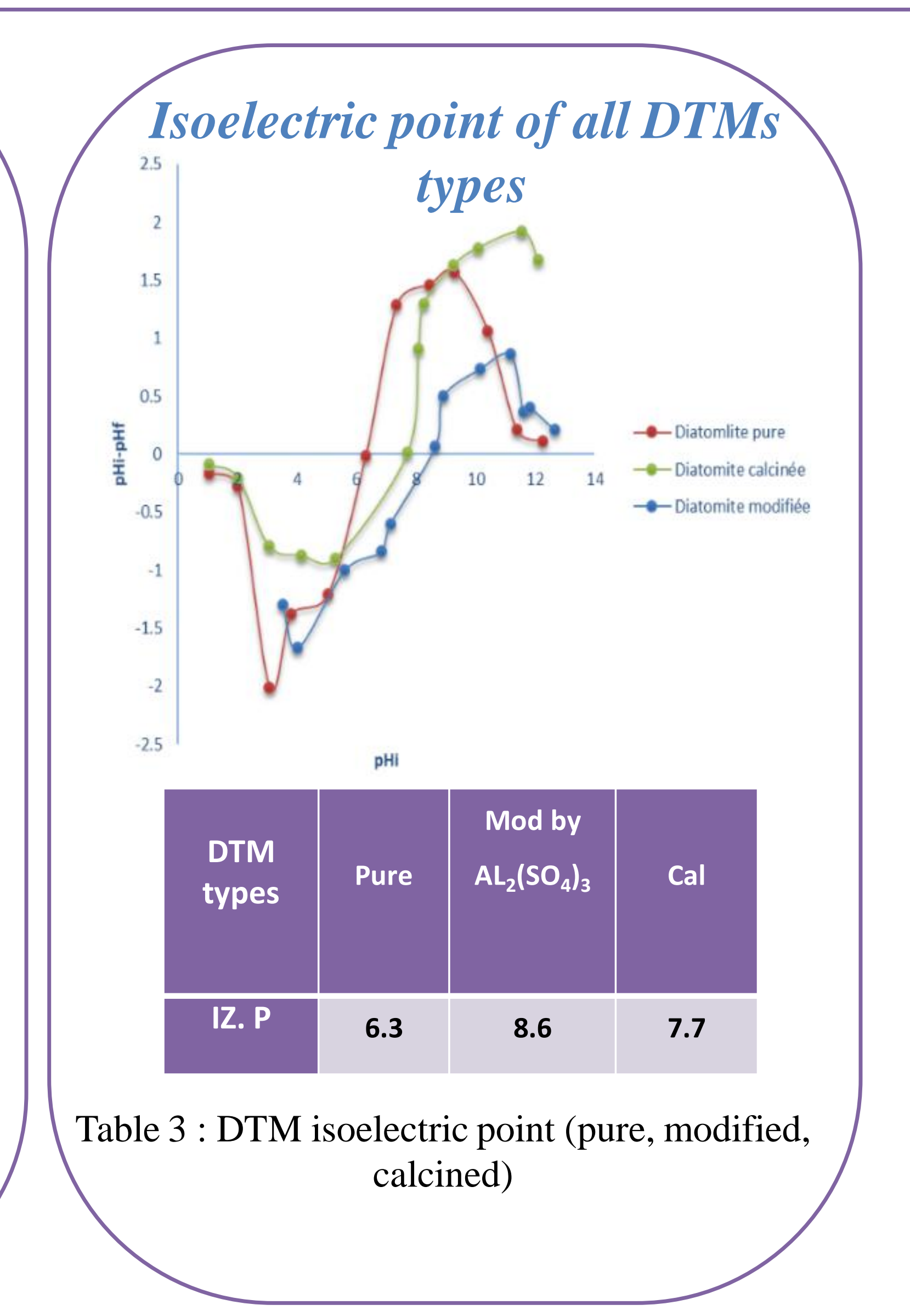

Resultis and discussion

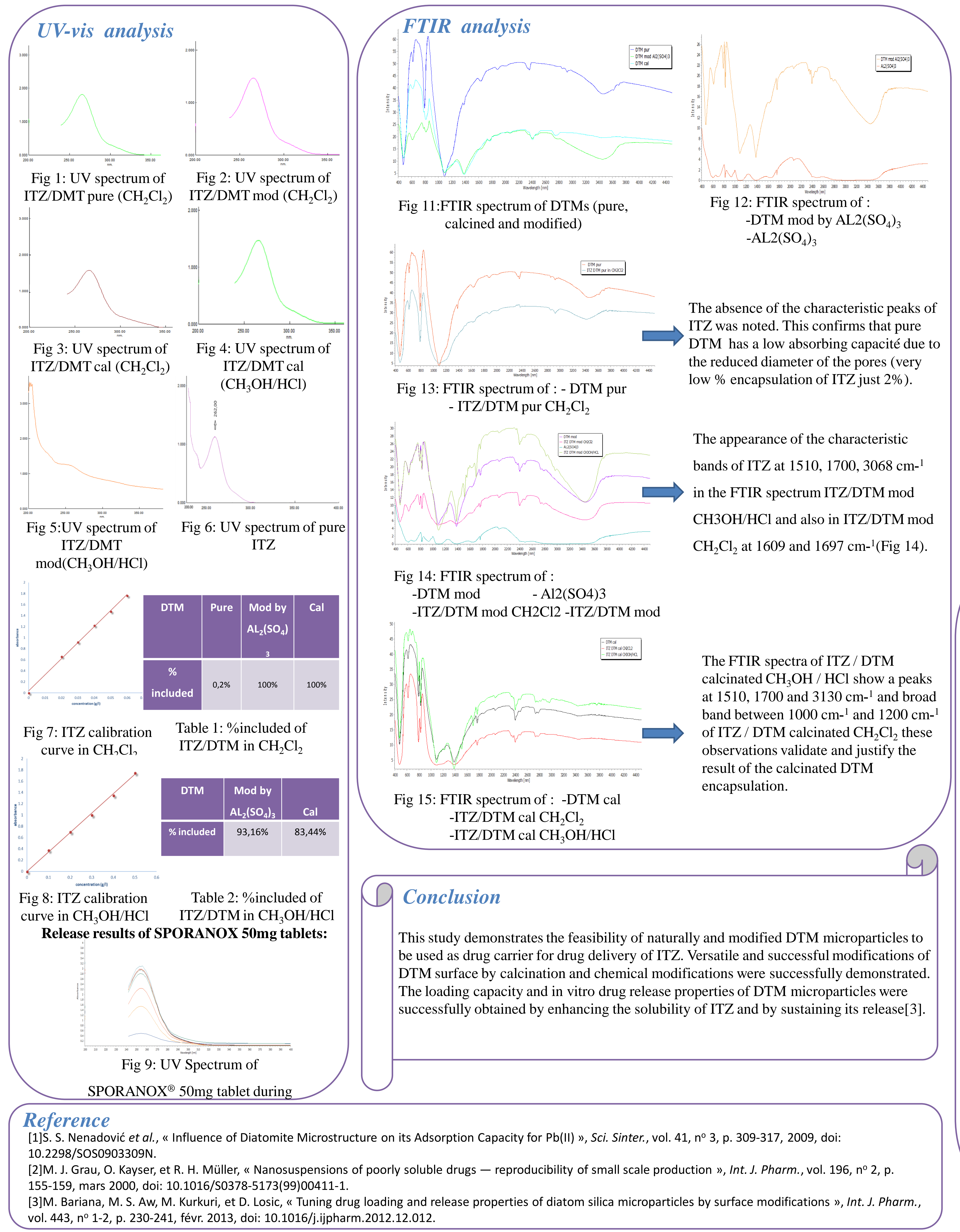

Optical microscopy analysis
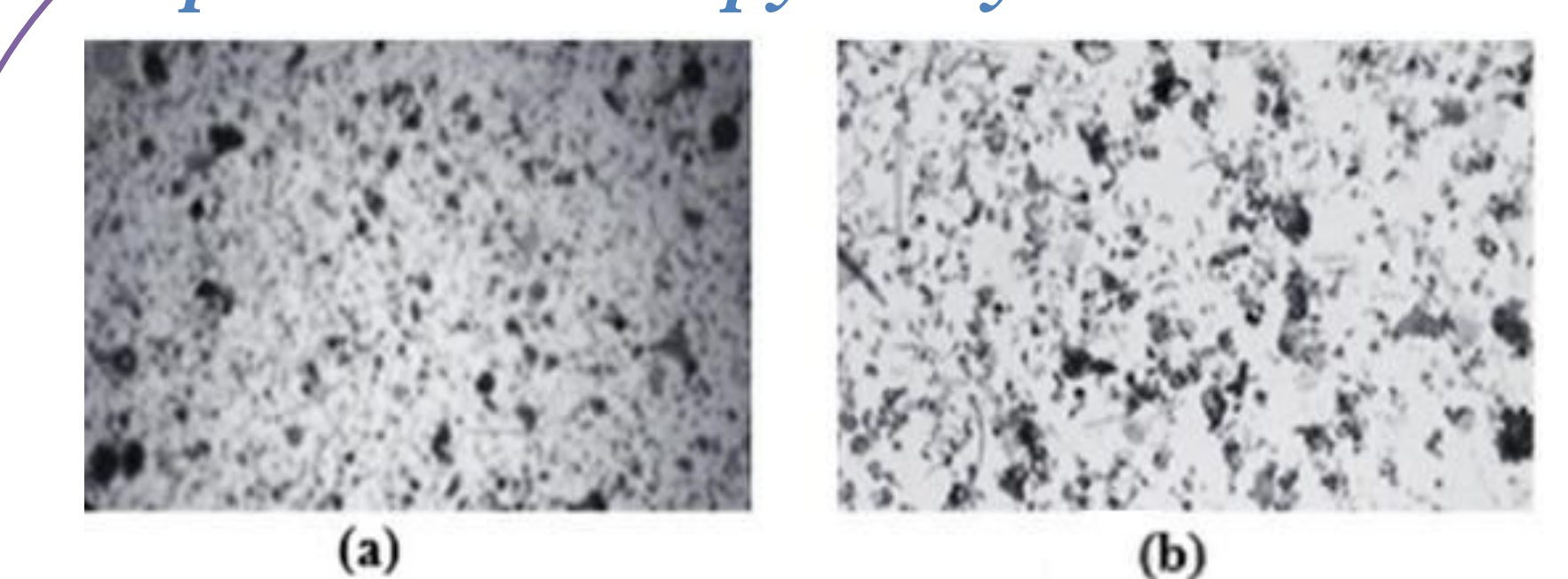

By comparing the pur DTM (a) with the chemically modified
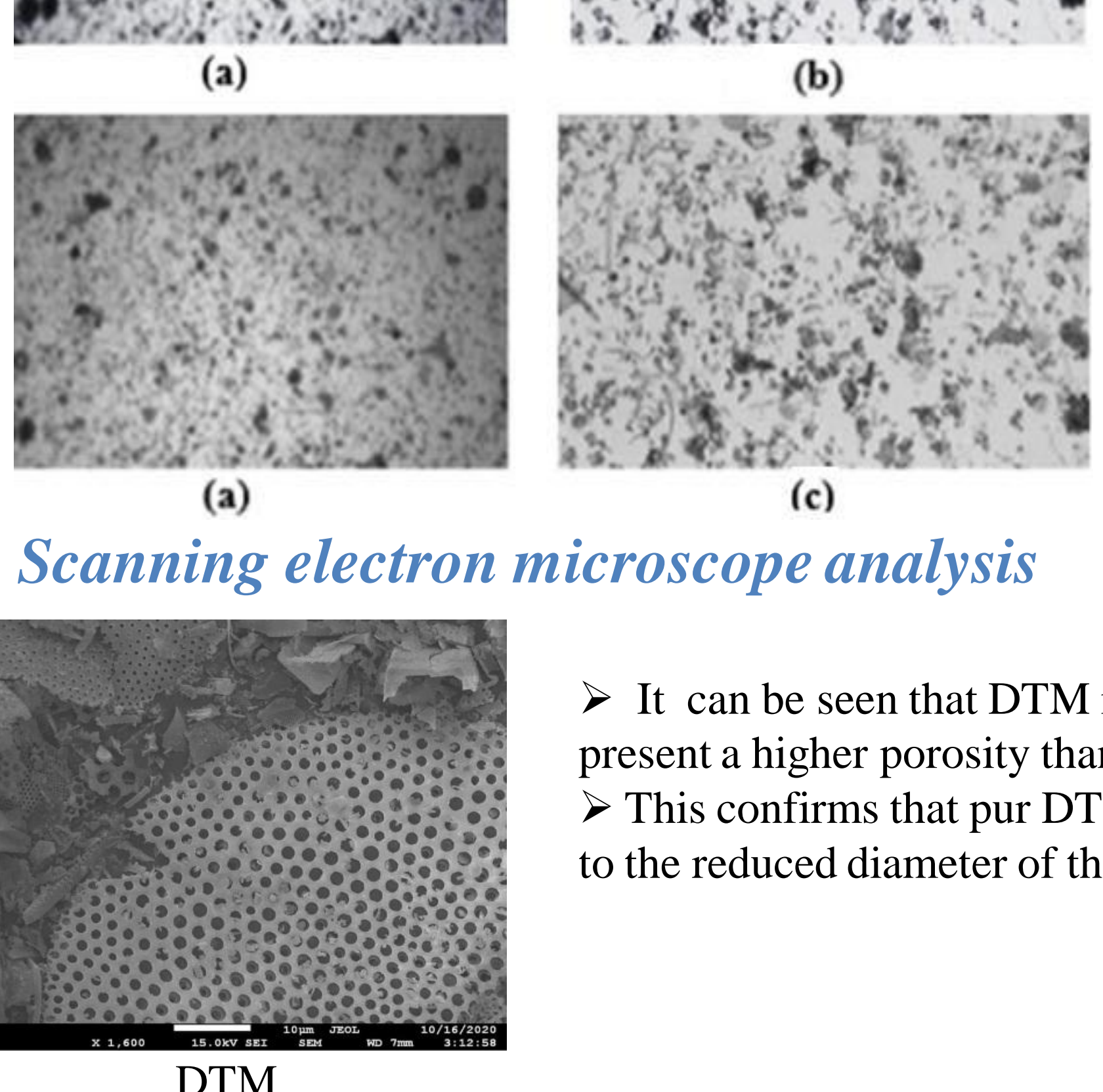
(b) and calcined (c)

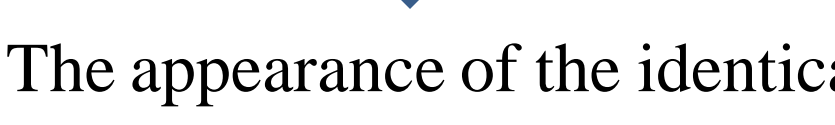
particle

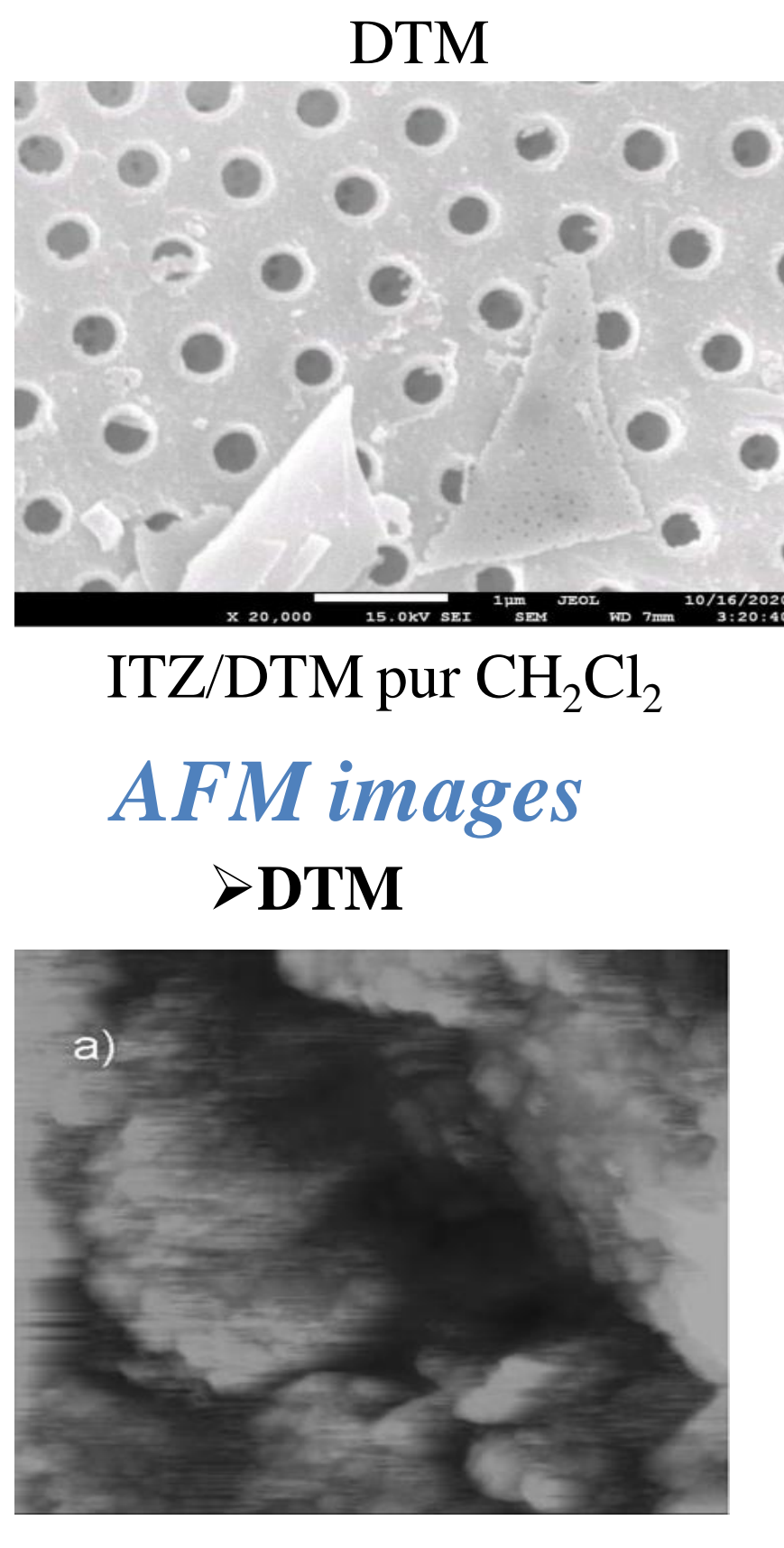

Fig 16: AFM image $(5 \times 5 \mu \mathrm{m})$ of DTM:
topography $[1]$ topography [1] present a higher porosity than DTM pur.

par encapsulate ITZ to the reduced diameter of the pores.
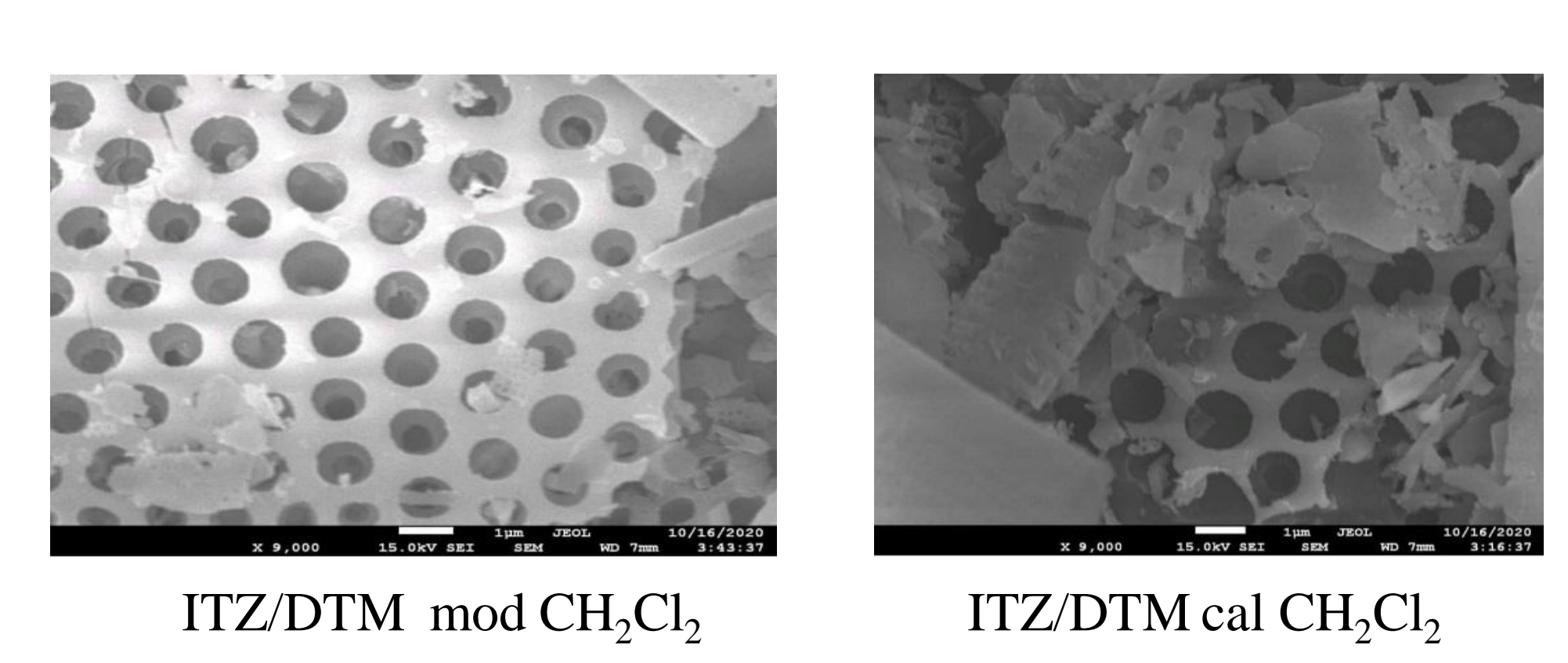

ITZ/DTM cal $\mathrm{CH}_{2} \mathrm{Cl}_{2}$

$>$ ITZ/DTM $\bmod \mathrm{CH}_{2} \mathrm{Cl}_{2}$

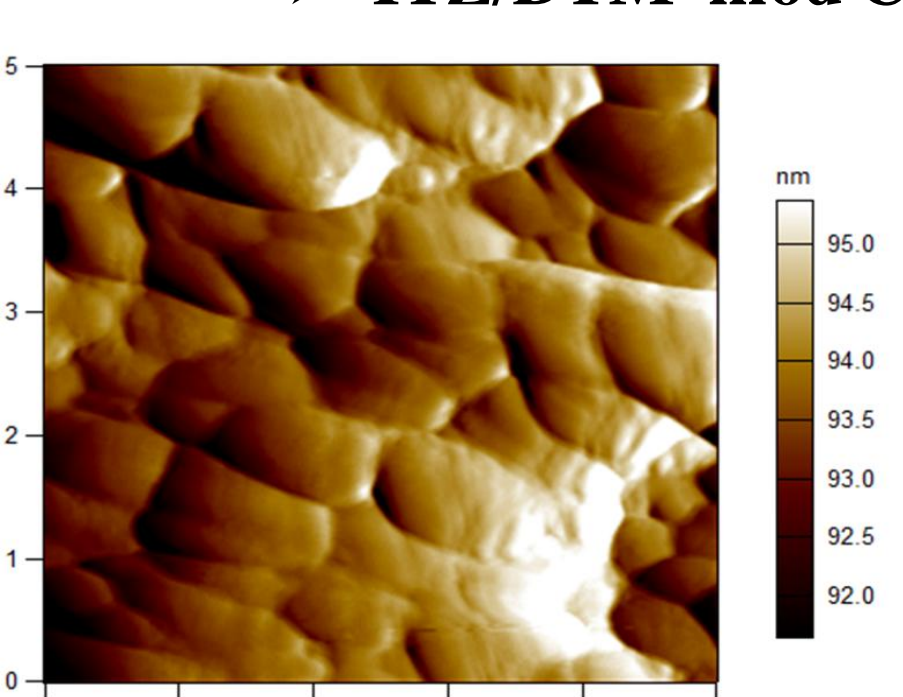

Fig 17: AFM image
(5x5 $\mathrm{mm})$ of ITZ/DTM $(5 \times 5 \mu \mathrm{m})$ of ITZ/D mod $\mathrm{CH}_{2} \mathrm{Cl}_{2}$ a)
topography.

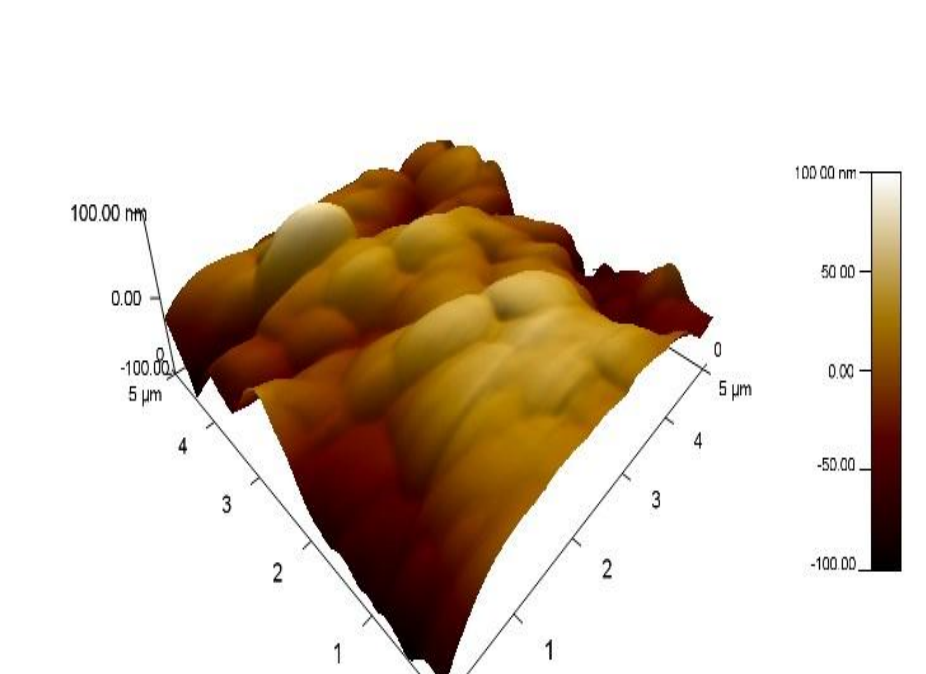

Fig 18: AFM 3D surface topography (500x500nm) of ITZ/DTM $\bmod \mathrm{CH}_{2} \mathrm{Cl}_{2}$

$>$ It can be seen from the fig 16 where the AFM micrographs of the diatomite are presented that small diatomite particles are compacted into the pellet. A sporge small pore sizes that can be noticed from topography picture [1] fig $17 . \quad \mathrm{RMS}=28.053 \mathrm{~nm}$

Release kinetics in the gastric meduim

Release kinetics in the gastric meduim

Physical mixture ITZ/DTM

ITZ/DTM mod complex

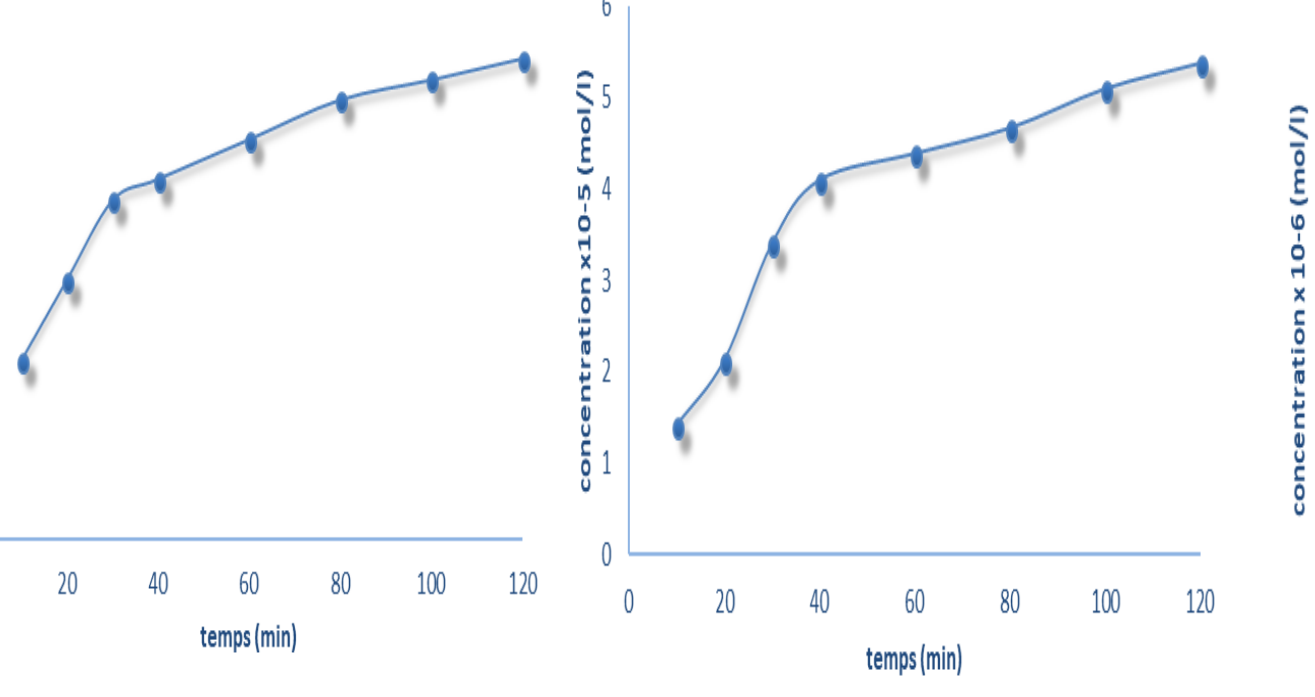

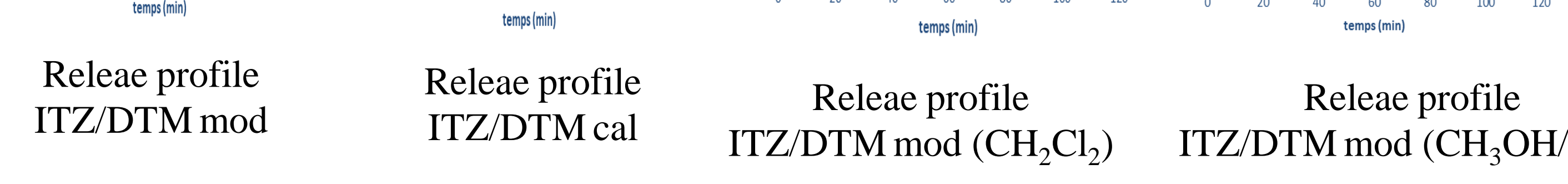
ITZ/DTM cal complex SPORANOX 50mg Tablet

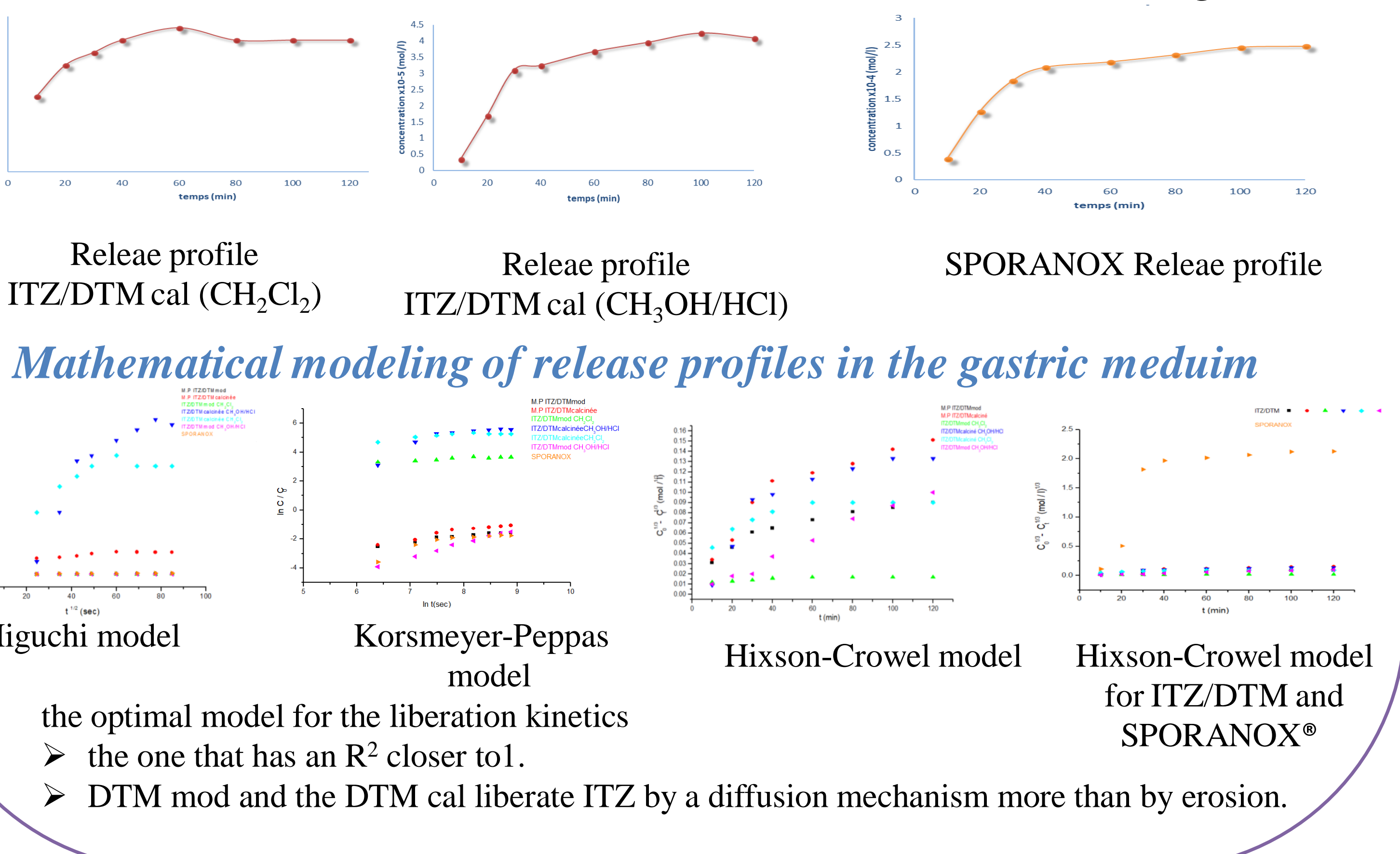

